\title{
High-cell-density regulation of the Pseudomonas aeruginosa type III secretion system: implications for tryptophan catabolites
}

Correspondence

Bertrand Toussaint

btoussaint@chu-grenoble.fr

Received 8 October 2007

Revised 15 May 2008

Accepted 22 May 2008
Da-Kang Shen, ${ }^{1}{ }^{2} \dagger$ Didier Filopon, ${ }^{1}+\ddagger$ Hichem Chaker, ${ }^{1}$ Stephanie Boullanger, ${ }^{3}$ Madiha Derouazi, ${ }^{1}$ Benoit Polack ${ }^{1}$ and Bertrand Toussaint ${ }^{1}$

\author{
${ }^{1}$ GREPI, TIMC-IMAG, UMR5525 CNRS/Université Joseph Fourier Faculté de Médecine, Bat. J \\ Roget, Domaine de la Merci, 38700 La Tronche, France \\ ${ }^{2}$ Department of Microbiology and Parasitology, Shanghai Jiao-Tong University School of Medicine, \\ Shanghai 200025, PR China \\ ${ }^{3}$ Service Spectrométrie de Masse, CERMAV-CNRS, BP53, 38041 Grenoble cedex 9, France
}

The Pseudomonas aeruginosa type III secretion system (T3SS) is known to be a very important virulence factor in acute human infections, but it is less important in maintaining chronic infections in which T3SS genes are downregulated. In vitro, the activation of T3SS expression involves a positive activating loop that acts on the transcriptional regulator ExsA. We have observed that in vivo T3SS expression is cell density-dependent in a manner that does not need known quorumsensing (QS) signals. In addition, stationary-phase culture supernatants added to exponentialphase growing strains can inhibit T3SS expression. The analysis of transposon insertion mutants showed that the production of such T3SS-inhibiting signals might depend on tryptophan synthase and hence tryptophan, which is the precursor of signalling molecules such as indole-3-acetic acid (IAA), kynurenine and Pseudomonas quinolone signal (PQS). Commercially available tryptophan-derived molecules were tested for their role in the regulation of T3SS expression. At millimolar concentrations, IAA, 1-naphthalacetic acid (NAA) and 3-hydroxykynurenine inhibited T3SS expression. Inactivation of the tryptophan dioxygenase-encoding kynA gene resulted in a decrease in the T3SS-inhibiting activity of supernatants. These observations suggest that tryptophan catabolites are involved in the downregulation of T3SS expression in the transition from a low- to a high-cell-density state.

\section{INTRODUCTION}

Pseudomonas aeruginosa is a Gram-negative opportunistic pathogen that causes acute and chronic infections in hospitalized individuals, burn victims and cystic fibrosis (CF) patients (Bodey et al., 1983; Lyczak et al., 2002; Pier, 2002). One of the major virulence factors of $P$. aeruginosa is the type III secretion system (T3SS), which consists of 43

†These authors contributed equally to this work.

$\ddagger$ Present address: URBM-FUNDP University of Namur, rue de Bruxelles 61, B-5000 Namur, Belgium.

Abbreviations: CF, cystic fibrosis; 2,4-D, dichlorophenoxyacetic acid; HSL homoserine lactone; IAA, indole-3-acetic acid; IBA, indole-3-butyric acid; LC-MS, liquid chromatography-MS; NAA, 1-naphthalacetic acid; PQS, Pseudomonas quinolone signal; OS, quorum-sensing; RLU, relative light units; T3SS, type III secretion system.

Two supplementary tables listing the roles of three Trp-derived signalling molecules and Pseudomonas spp. genes encoding enzymes involved in IAA biosynthesis are available with the online version of this paper. coordinately regulated genes encoding type III secretion and translocation machinery, regulatory factors, type III effectors and effector-specific chaperones (Yahr \& Wolfgang, 2006). The T3SS is used to deliver a unique set of effectors directly into the cytoplasm of infected host cells that subvert normal host cell physiology in order to promote pathogenesis (Hueck, 1998). Epidemiological studies of $P$. aeruginosa isolated from patients have shown that the presence of a functional T3SS is strongly associated with a higher incidence of systemic spread and a poor clinical outcome (Garau \& Gomez, 2003; Hauser et al., 2002; Roy-Burman et al., 2001). To date, four effectors have been described in P. aeruginosa, ExoS, ExoT, ExoY and ExoU. Curiously, most strains of $P$. aeruginosa possess either the gene for ExoS or the gene for ExoU, but not both (Feltman et al., 2001). In this study, we have used the CHA strain, whose genome contains the genes encoding ExoS, ExoT and ExoY, but not ExoU (Dacheux et al., 1999; DelicAttree et al., 1995). T3SS expression is induced by at least 
three different environmental elements: (i) in vivo contact with eukaryotic host cells; (ii) in vitro calcium depletion in the medium; and (iii) the presence of serum (Vallis et al., 1999; Yahr \& Frank, 1994).

All T3SS genes are coordinately regulated by ExsA, an AraC-like master transcriptional activator, which binds to a consensus sequence in the promoter region of these genes (Hovey \& Frank, 1995). In recent years, it has been discovered that a cascade of T3SS proteins, including ExsD, ExsC and ExsE, influences the activity of ExsA (Dasgupta et al., 2004; McCaw et al., 2002; Rietsch et al., 2005; Urbanowski et al., 2005). It is interesting to note that $\operatorname{exs} A$, exs $C$ and exsE are located in the same operon, exsCEBA, which is positively controlled by ExsA and PsrA (Shen et al., 2006). ExsE is a secreted regulator of T3SS. When the secretion channel is closed, ExsE is complexed with its chaperone ExsC in the cytoplasm, and the transcription of the T3SS genes is repressed due to the sequestration of ExsA by ExsD, an anti-activator. When the secretion channel is opened, ExsE is secreted, leaving ExsC free to interact with ExsD, which releases ExsA, thereby allowing liberated ExsA to activate the transcription of the T3SS genes (including the exsCEBA operon). This process represents the positive activation loop of the T3SS that is triggered by cell contact in vivo and can be mimicked by calcium depletion in vitro. Besides this major regulatory operon, other factors regulate T3SS expression, including cyclic AMP with Vfr, the Pseudomonas catabolite repression homologue (Wolfgang et al., 2003), PtrA (Ha et al., 2004) and the RetS/LadS/GacAS two-component regulatory systems (Goodman et al., 2004; Laskowski et al., 2004; Ventre et al., 2006; Zolfaghar et al., 2005). Taken together, these published data indicate that $P$. aeruginosa uses a complex set of signalling pathways both to activate and to repress T3SS expression in response to extracellular and intracellular triggers (Yahr \& Wolfgang, 2006).

Several reports suggest that a functional $P$. aeruginosa T3SS is far less common in CF patient isolates than in isolates from patients with acute infections (Dacheux et al., 2000; Roy-Burman et al., 2001). It has been proposed that, following the infection of CF patient airways, $P$. aeruginosa strains evolve to reduce T3SS expression (Lee et al., 2005), or that populations of cells gradually change from a type III protein secretion-positive phenotype to a secretion-negative phenotype (Jain et al., 2004). This pattern seems to be common in bacterial infection, since other T3SSs are downregulated during mammalian cell infection by Salmonella enterica serovar Typhimurium, Escherichia coli O157 and Shigella flexneri (Dahan et al., 2004; Eriksson et al., 2003; Faucher et al., 2006; Lucchini et al., 2005). The consistency of these observations suggests that the downregulation of T3SS expression might be a conserved phenomenon that follows the successful infection of mammalian cells by these Gram-negative pathogens. Presumably, a mechanism exists for the inactivation of T3SS expression once it has served its purpose. This is the case in enterohaemorrhagic and enteropathogenic E. coli, in which maximum T3SS expression occurs during the transition from the late exponential to the stationary phase (Sperandio et al., 1999), but little is known about the mechanisms involved in the arrest of the positive activation loop of the $P$. aeruginosa T3SS.

In this study, we observed that T3SS expression was repressed by stationary-phase culture supernatants, including those from the $P$. aeruginosa wild-type strains CHA or $\mathrm{PAO} 1$ and those from mutants that do not produce known quorum-sensing (QS) signals, such as 3-oxo-C12 and $\mathrm{C} 4$ acylhomoserine lactone (3-oxo- $\mathrm{C}_{12}-\mathrm{HSL}$ and $\mathrm{C}_{4}$ - $\mathrm{HSL}$ ) or the Pseudomonas quinolone signal (PQS). We then investigated the nature of the T3SS-inhibiting signal(s) produced in the stationary-phase culture supernatants and showed that the production of such regulatory signal(s) may depend on the catabolism of tryptophan (Trp), which is the precursor of several important signalling molecules, including indole-3-acetic acid (IAA), kynurenine and PQS.

\section{METHODS}

Bacterial strains and plasmids. All bacterial strains and plasmids used in this study are listed in Table 1. P. aeruginosa strains were maintained on Pseudomonas Isolation Agar (Difco) or cultivated in Luria broth (LB) with antibiotics if required [300 $\mu \mathrm{g}$ carbenicillin $\mathrm{ml}^{-1}, 200 \mu \mathrm{g}$ gentamicin $(\mathrm{Gm}) \mathrm{ml}^{-1}$ and $250 \mu \mathrm{g}$ tetracycline $\mathrm{ml}^{-1}$. E. coli strains were cultivated in LB with antibiotics if required $\left(100 \mu \mathrm{g}\right.$ ampicillin $\mathrm{ml}^{-1}, 10 \mu \mathrm{g} \mathrm{Gm} \mathrm{ml} \mathrm{gm}^{-1}$ and $20 \mu \mathrm{g}$ tetracycline $\left.\mathrm{ml}^{-1}\right)$. P. aeruginosa strains were stored in Protect bacterial preservers (TSC) at $-80{ }^{\circ} \mathrm{C}$. E. coli strains were maintained at $-80{ }^{\circ} \mathrm{C}$ in $50 \%(\mathrm{v} / \mathrm{v})$ glycerol stocks. To induce T3SS expression, $P$. aeruginosa strains were grown in LB supplemented with $5 \mathrm{mM}$ EGTA and $20 \mathrm{mM} \mathrm{MgCl}_{2}$ (T3SS-inducing conditions). T3SS non-inducing conditions were $\mathrm{LB}$ with $5 \mathrm{mM} \mathrm{CaCl}_{2}$.

Mutant construction. All PCR primers employed in this study are listed in Table 2 and are based on the PAO1 genome sequence (http:// www.pseudomonas.com) (Stover et al., 2000). All amplifications were carried out with Pfu Turbo polymerase (Promega) by using CHA genomic DNA as a template. PCR products were subcloned into pTOPO (Invitrogen) and sequenced to confirm that no mutations had been introduced during amplification. Plasmids were transformed into chemically competent $\mathrm{DH} 5 \alpha$ E. coli. All mutant $P$. aeruginosa strains made in this study were derived from wild-type CHA (Toussaint et al., 1993). Unmarked mutants were constructed by removing an internal fragment of coding sequence by means of allelic exchange (Schweizer \& Hoang, 1995), using a Cre-lox antibiotic marker recycling method (Quenee et al., 2005). Briefly, the upstream and downstream regions flanking the gene to be deleted were amplified using the primer pairs indicated in Table 2. The amplified regions were subcloned in tandem into the gene replacement vector pEX100Tlink (Quenee et al., 2005). Next, the Gmlox resistance marker was inserted between the two fragments to generate the vector pKO-gene as indicated in Table 1. These constructs were then transformed into E. coli S17-1 and mobilized into CHA, CHA carrying an exsCEBA promoter-lux fusion ( $\mathrm{pC}:: \operatorname{lux}$ ) or CHA carrying an exoS promoter-lux fusion ( $\mathrm{pS}:$ : lux) (Shen et al., 2006) by mating. Gm-resistant transformants were isolated, the Gmresistance gene was then excised and gene deletion was confirmed by PCR with the primers indicated in Table 2 . The resulting resistancemarker-free mutants, shown in Table 1 , had no discernible growth differences when compared with wild-type CHA. 
Table 1. Bacterial strains and plasmids used in this study

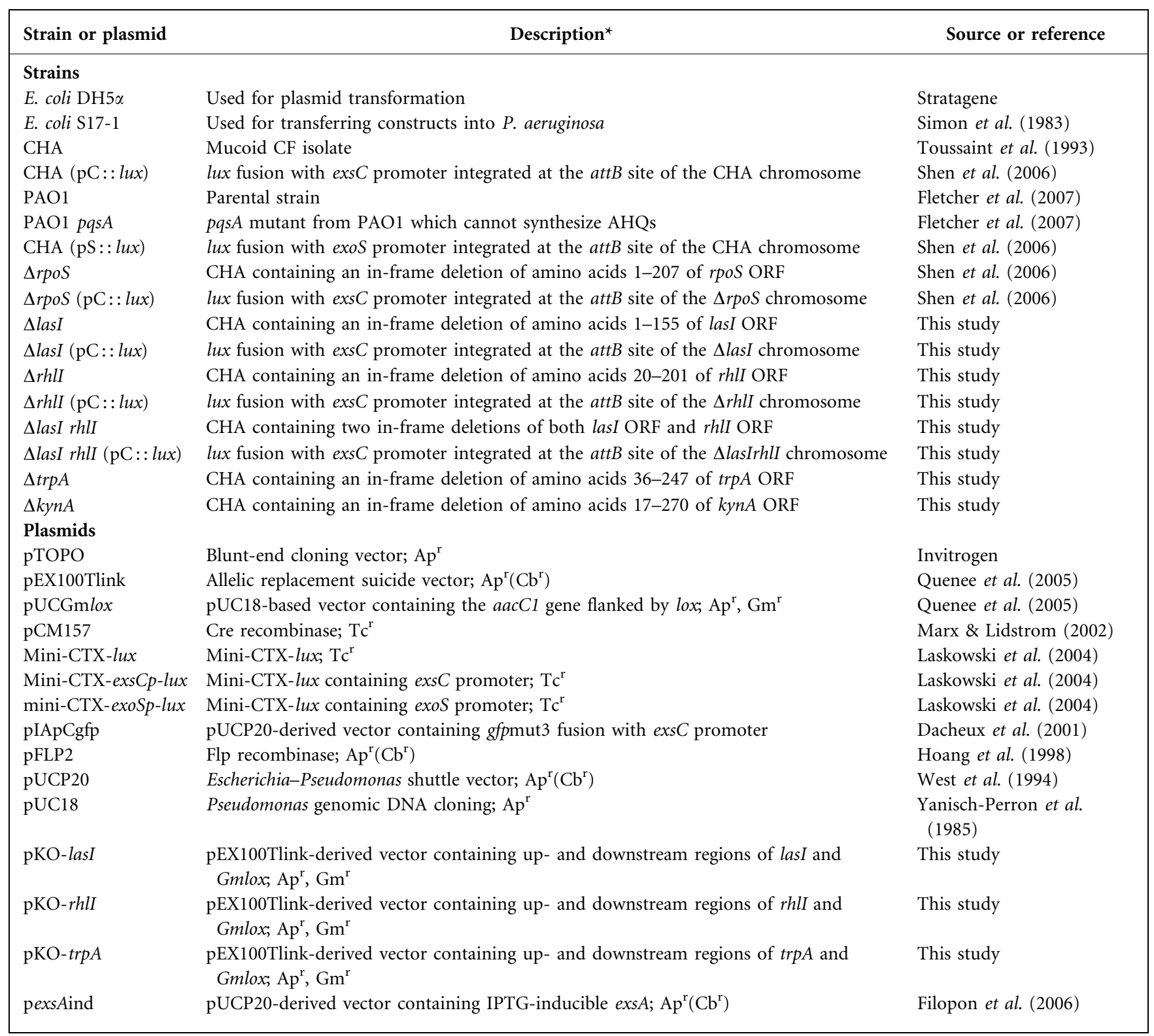

${ }^{\star} \mathrm{Ap}^{\mathrm{r}}$, ampicillin resistance; $\mathrm{Cb}^{\mathrm{r}}$, carbenicillin resistance; $\mathrm{Gm}^{\mathrm{r}}, \mathrm{Gm}$ resistance; $\mathrm{Tc}^{\mathrm{r}}$, tetracycline resistance.

Preparation of the stationary-phase culture supernatants. Overnight cultures of bacteria were washed twice with LB, reinoculated at $1: 100$ in $100 \mathrm{ml} \mathrm{LB}$ and grown at $37{ }^{\circ} \mathrm{C}$ with agitation at 260 r.p.m. to $\mathrm{OD}_{600} 2.5-3$ (stationary phase, high cell density). Bacteria were harvested by centrifugation at $8000 \mathrm{~g}$ for $10 \mathrm{~min}$ at room temperature, then washed briefly with $10 \mathrm{ml}$ VogelBonner medium (VB) (Vogel \& Bonner, 1956) and grown overnight in $100 \mathrm{ml} \mathrm{VB}$ at $37{ }^{\circ} \mathrm{C}$ with agitation. Supernatants were collected by centrifugation at $15000 \mathrm{~g}$ for $20 \mathrm{~min}$ at $4{ }^{\circ} \mathrm{C}$ and stored at $-80{ }^{\circ} \mathrm{C}$. To obtain concentrated samples, supernatants were prepared normally, lyophilized by freeze dry system/Lyph Lock 4.5 (Labconco), resuspended in a small volume of water, precipitated by incubation in 3 volumes of ethanol for $1 \mathrm{~h}$ at $-20^{\circ} \mathrm{C}$, and centrifuged at $15000 \mathrm{~g}$ at $4{ }^{\circ} \mathrm{C}$ for $45 \mathrm{~min}$ to remove salts and proteins. Alcohol was removed by the SpeedVac system (Thermo) or a Rotavapour evaporator (Buchi), and the precipitate was redissolved in water to obtain the desired concentration, calculated relative to the starting volume of supernatant. This treatment did not change the activity of supernatants.

Reporter gene analysis. Bacterial strains containing $(\mathrm{pC}:: \operatorname{lu} x)$ or (pS::lux) were grown in LB with aeration at $37^{\circ} \mathrm{C}$ for $14-16 \mathrm{~h}$. Bacteria were washed with $\mathrm{LB}$ and then cultured from $\mathrm{OD}_{600} 0.05$ in LB under T3SS-inducing or non-inducing conditions. The stationaryphase culture supernatants and commercial molecules, including Trp (up to $2.5 \mathrm{mM}$ ), IAA sodium salt, indole-3-butyric acid (IBA) potassium salt, 1-naphthalacetic acid (NAA) potassium salt, 2,4dichlorophenoxyacetic acid (2,4-D) potassium salt, and kynurenine sulfate salt or 3-hydroxykynurenine (Sigma), were then added to the culture in order to determine their roles in the regulation of T3SS 
Table 2. Primer sequences used in this study

\begin{tabular}{|c|c|c|}
\hline Primer & Sequence $^{*}$ & Description \\
\hline lasI-U1 & 5'-ACGGATCCAAGTGGCTATGTCGCCG-3' & Amplification of upstream region flanking lasI \\
\hline lasI-U2 & 5'-ACAAGCTTCCGGACCCTTGCTAGGC-3' & \\
\hline lasI-D1 & 5'-AGAAGCTTGGCCTGGACGTATCGCG-3' & Amplification of downstream region flanking lasI \\
\hline lasI-D2 & 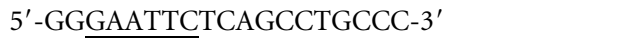 & \\
\hline rhlI-U1 & 5'-TATCTAGACGCGGTGCGCCGCAAGG-3' & Amplification of upstream region flanking rhlI \\
\hline rhlI-U2 & 5'-ATAAGCTTGCTCGGCGATCATGGCG-3' & \\
\hline rhll-D1 & 5'-ATAAGCTTGCGTCATCGGGCGTTCC-3' & Amplification of downstream region flanking rhlI \\
\hline rhlI-D2 & 5'-CAGAGCTCTGTCCGGAAATCCTCATGC-3' & \\
\hline $\operatorname{trp} A-\mathrm{U} 1$ & 5'-AGAATTCTCAGATCAGCTCGTGGCGG-3' & Amplification of upstream region flanking $\operatorname{trp} A$ \\
\hline $\operatorname{trp} A-\mathrm{U} 2$ & 5'-TAAGCTTCAGGCGGTCAAGGATGTCCT-3' & \\
\hline $\operatorname{trp} A-\mathrm{D} 1$ & 5'-TAAGCTTCCAGGGAAGACGCGTAGT-3' & Amplification of downstream region flanking $\operatorname{trp} A$ \\
\hline $\operatorname{trp} A-\mathrm{D} 2$ & 5'-TGGATCCGCACAAGATCAACAACTGC-3' & \\
\hline kynA-U1 & 5'-AGAGCTCTGCTCAACGGCGGAAAGCGTT-3' & Amplification of upstream region flanking kynA \\
\hline kynA-U2 & 5'-TAAGCTTCTCTCCGTCCGTTTCTCCCTG-3' & \\
\hline kynA-D1 & 5'-TAAGCTTAGCATGCTCGATACCATCCTC-3' & Amplification of downstream region flanking $k y n A$ \\
\hline kynA-D2 & 5'-AGGATCCATCGACCAACGCCTGATCAA-3' & \\
\hline lasI-1 & 5'-GTGGATGCTCAAGAAGGACTAC-3' & Verification of $\Delta$ lasI \\
\hline lasI-2 & 5'-CTGATCGGCAACCTTACCC-3' & \\
\hline rhlI-1 & 5'-CGCGGCGCTGGGTCTCATC-3' & Verification of $\Delta r h l I$ \\
\hline rhll -2 & 5'-GTAACCGCCCTCTTCCGTGCGG-3' & \\
\hline $\operatorname{trp} A-1$ & 5'-TCAGCGTGCGTTGCGCACGC-3' & Verification of $\Delta \operatorname{trp} A$ \\
\hline $\operatorname{trp} A-2$ & 5'-AAGCATGAGCCGCCTGCAGA-3' & \\
\hline kynA-1 & 5'-TACCGTCGCTGCCTACCAGGC-3' & Verification of $\Delta k y n A$ \\
\hline kynA-2 & 5'-CATGGGGCTTTTGCGGCAAGA-3' & \\
\hline $\operatorname{aacCl-F}$ & 5'-ATGTTACGCAGCAGCAACGA-3' & Amplification of $a a c C 1$ probe for Southern blotting \\
\hline aacC1-R & 5'-CAAGTTCCCGAGGTAATCGG-3' & \\
\hline
\end{tabular}

${ }^{\star}$ Restriction enzyme sites are underlined.

gene expression and toxin secretion. Supernatant was diluted to $1 \times$ from concentrated stationary-phase supernatant, and commercial molecules were used at $1 \mathrm{mM}$ concentration. Relative luminescence units (RLU) of $0.2 \mathrm{ml}$ of culture were measured using a Luminoskan Ascent spectrophotoluminometer (Labsystems) or a TrisStar LB 941 spectrophotoluminometer (Berthold Technologies) for data with $\mathrm{PAO} 1, \mathrm{PAO} 1$ pqsA and the kynA mutant strains. Bacterial quantity was determined by measuring the $\mathrm{OD}_{600}\left(\mathrm{OD}_{600}\right.$ of 1 corresponds to $6 \times 10^{8}$ bacteria $\mathrm{ml}^{-1}$ under our growth conditions). The transcription level was expressed as the number of RLU per $6 \times 10^{8}$ bacteria, as indicated. The means and SE presented were obtained from the data for at least three independent experiments each done in triplicate on different days using different starting cultures. To test the possible effects of nutrient deprivation on T3SS activation, CHA (pIApCgfp) was grown from $\mathrm{OD}_{600} 0.05$ in LB (main culture). At different growth times, aliquots were taken and diluted with fresh $\mathrm{LB}$ to $\mathrm{OD}_{600} 0.1$, or the $\mathrm{OD}_{600}$ of the main culture was maintained and the medium was replaced with fresh LB. Bacteria were then induced to express T3SS genes for $3 \mathrm{~h}$ or were left uninduced. The level of exsCEBA transcription was measured by fluorescence and expressed as the fold-induction of the $\mathrm{pC}$ promoter (induced level/non-induced level). The calculated means and SE were obtained from data from three independent experiments.

To measure the T3SS transcription level in the presence of human $\mathrm{AB}$ serum, overnight bacterial cultures were washed with LB, precultured at $1: 100$ in LB to $\mathrm{OD}_{600} 0.6-1$, washed twice with modified HEPESbuffered saline (mHBS) (Dacheux et al., 1999), and plated at $5 \times 10^{5}$ cells per well in a 96-well culture plate with or without $10 \%$ human serum. RLU readings from the entire culture plate were taken immediately after the addition of bacteria [time $(t)=0$ ] and at $1 \mathrm{~h}$ intervals.

Secretion profile analysis. To examine the effect of IAA and NAA on type III effector secretion, we used wild-type CHA containing the IPTG-inducible plasmid pexsAind (Filopon et al., 2006), which could express exogenous ExsA in trans. Bacterial strains were cultivated overnight in LB containing appropriate antibiotics at $37{ }^{\circ} \mathrm{C}$. These cultures were washed twice with $\mathrm{LB}$, reinoculated at $1: 100$ in $\mathrm{LB}$ supplemented with different compounds, and then vigorously shaken at $37{ }^{\circ} \mathrm{C}$ for $3 \mathrm{~h}$. Supernatants of the bacterial culture (equivalent to $1 \mathrm{ml}$ at $\mathrm{OD}_{600} 1$ ) were collected and TCA was added to $13 \%$ to precipitate proteins at $4{ }^{\circ} \mathrm{C}$ for $30 \mathrm{~min}$. The precipitated proteins were collected by centrifugation at $15000 \mathrm{~g}$ for $15 \mathrm{~min}$, washed twice with cold acetone, air-dried, resuspended in SDS-PAGE loading buffer, analysed by $10 \%$ SDS-PAGE and visualized by Coomassie staining. Western blots of normalized secreted proteins or of total bacterial lysates were obtained with the use of an anti-ExoS/ExoT serum at $1: 1000$ dilution.

Transposon mutagenesis, screening and characterization. A library of transposon insertion mutants was generated by mobilizing the suicide plasmid pFAC from E. coli S17-1 to P. aeruginosa wildtype CHA, as described elsewhere (Wong \& Mekalanos, 2000). Briefly, two strains growing at exponential phase were mixed, placed on PIA plates and incubated overnight at $37^{\circ} \mathrm{C}$. Bacteria were resuspended in LB at appropriate dilutions, which were then spread on PIA containing Gm. Approximately 2000 Gm-resistant mutants, from a 
library of $\sim 10^{6}$ clones, were tested. Mutants were grown on microplates and centrifuged, and each supernatant was tested for its ability to inhibit exoS expression using the CHA (pC::lux) reporting strain grown in a microplate. To avoid artefacts due to microplate culture conditions, 20 insertion mutants whose supernatants were inactive during the first screen were tested in a second round by conventional culture in $15 \mathrm{ml}$ aerated tubes. Two mutants of the 20 fulfilled the requirement for further analysis (no inhibition of T3SS activation of wild-type control strain). These two mutants were further characterized by cloning all PstI-digested genomic DNA into plasmid pUC18 with Gm selection and then by sequencing the PstI fragments with M13 forward or reverse primers. Southern blot hybridization was performed with a DIG Nucleic Acid Detection kit (Roche) to verify the number of insertion sites in the genomic DNA.

Liquid chromatography-MS (LC-MS) analysis. To assess the level of known QS signals produced by the wild-type strain CHA and its QS mutants, the stationary-phase culture supernatants were quantified by LC-MS analysis of dichloromethane extracts, as described previously (Morin et al., 2003).

\section{RESULTS}

\section{T3SS expression is dependent on cell density}

Chromosomal transcriptional gene reporters composed of an exsCEBA promoter-lux fusion (pC::lux), or an exoS promoter-lux fusion ( $\mathrm{pS}:: \operatorname{lu} x)$ were used to identify the transcriptional level of T3SS genes (Shen et al., 2006) during bacterial growth. From the results shown in Fig. 1(a) it appears that both exsCEBA and exoS were expressed in a growth phase-dependent manner when the strains were initially cultured from $\mathrm{OD}_{600} 0.05$ (low cell density) in T3SS-inducing conditions. The expression of exsCEBA and exoS rapidly attained its maximum levels at $\mathrm{OD}_{600} \sim 1.7$ and $\sim 2$, respectively; it then began to decrease with increasing $\mathrm{OD}_{600}$ (high cell density). To test for the possible effects of nutrient deprivation during culture growth, i.e. its possible responsibility for exsCEBA and exoS repression, in vitro T3SS activation was tested on cultures at a different cell densities in fresh LB medium to avoid the effects of nutrient deprivation. At different growth times, aliquots of a CHA (pIApCgfp) culture (main culture) were taken, the medium was replaced with fresh LB and the cell density was maintained or set to 0.1 . Bacteria were then induced to express T3SS genes or were left uninduced. The level of exsCEBA transcription was measured by fluorescence and expressed as the fold-induction of the $\mathrm{pC}$ promoter. T3SS repression was still observed in high-celldensity cultures $\left(\mathrm{OD}_{600}>2\right)$ even in fresh media, unlike in bacteria diluted to low cell density before T3SS induction (Fig. 1c). Thus, the effect of nutrient deprivation on the transcription of T3SS genes could not be responsible for the phenotype that we observed at high cell density for the selected T3SS genes. Cell density-dependent repression of this virulence system has been reported elsewhere for $P$. aeruginosa strain PAK but not further investigated ( $\mathrm{Ha} \&$ Jin, 2001). Moreover, such a decline in the expression of exsCEBA and exoS over time was reminiscent of QS regulation, in which a sufficient number of bacteria (the bacterial 'quorum') initiate the activation or the repression of multiple genes (Fuqua et al., 1994). The fact that QS has an effect on T3SS expression in P. aeruginosa (Bleves et al., 2005; Hogardt et al., 2004; Juhas et al., 2005; Schaber et al., 2004) prompted us to examine the possibility that the reduction of exsCEBA and exoS expression over time was due to QS-based regulation, which controls gene expression in a cell density-dependent manner through the production of different QS-signalling molecules.

\section{Stationary-phase culture supernatants, as opposed to QS signals, inhibit exsCEBA expression in vitro}

We constructed mutants known to be deficient in QS signal generation (Juhas et al., 2005): CHA $\Delta$ lasI, CHA $\Delta$ rhlI and CHA $\triangle$ lasI-rhlI, and T3SS expression was examined during bacterial growth. First, these mutants showed the same pattern as the wild-type CHA strain: the expression of the exsCEBA operon rapidly attained its maximum level at $\mathrm{OD}_{600} \sim 1.7$ and then began to decrease with increasing $\mathrm{OD}_{600}$ (Fig. 1b). We also observed, independent of the growth stage, a higher activity from the promoter of the exsCEBA operon, $\mathrm{pC}$, in the $\Delta$ rhlI mutant and the $\Delta$ lasIrhlI double mutant than in the CHA strain. This last result is in agreement with earlier studies showing inhibition of the T3SS by the Rhl QS system (Bleves et al., 2005; Hogardt et al. 2004). Second, we examined the ability of the wildtype and mutant strains to produce and secrete a T3SS inhibitory signal at high cell density. To do so, stationaryphase culture supernatants were prepared from the different strains as described in Methods. Then, to evaluate their influence on the expression of exsCEBA, each supernatant was added to the CHA ( $\mathrm{pC}:: \operatorname{lux}$ ) strain, which was cultured from an initial $\mathrm{OD}_{600}$ of 0.05 in $\mathrm{LB}$ under T3SS-inducing conditions. The transcriptional activity from $\mathrm{pC}$ in the presence of supernatants from either the wild-type or the QS mutants was roughly twofold lower than in the absence of supernatants (Fig. 1d). As control, the same volume of VB was added instead of supernatant, and no inhibitory effect on T3SS expression was observed, ruling out a role for $\mathrm{VB}$ medium in the repression phenomenon. In order to exclude the role of known $P$. aeruginosa QS signal molecules in T3SS inhibition, stationary-phase culture supernatants were analysed by LC-MS. As shown in Table 3, both 3-oxoC12-HSL and C4-HSL were produced in the wild-type CHA stain; conversely, the respective acylhomoserine lactone (AHL) was absent in the mutants $\Delta l a s I$ and $\Delta r h l I$ and in the double mutant slasI-rhlI. Furthermore, extraction experiments on wild-type stationary-phase supernatants using methods known to extract QS signals showed that the T3SS inhibitor(s) was not found in the organic phase, unlike the classical QS molecules (data not shown). In order to study the role of the third known QS signal molecule in $P$. aeruginosa, $\mathrm{PQS}$, strains $\mathrm{PAO} 1$ and PAO1 pqsA were used. The $p q s A$ mutant is not able to produce hydroxyl-alkyl-quinolone signals, including PQS 

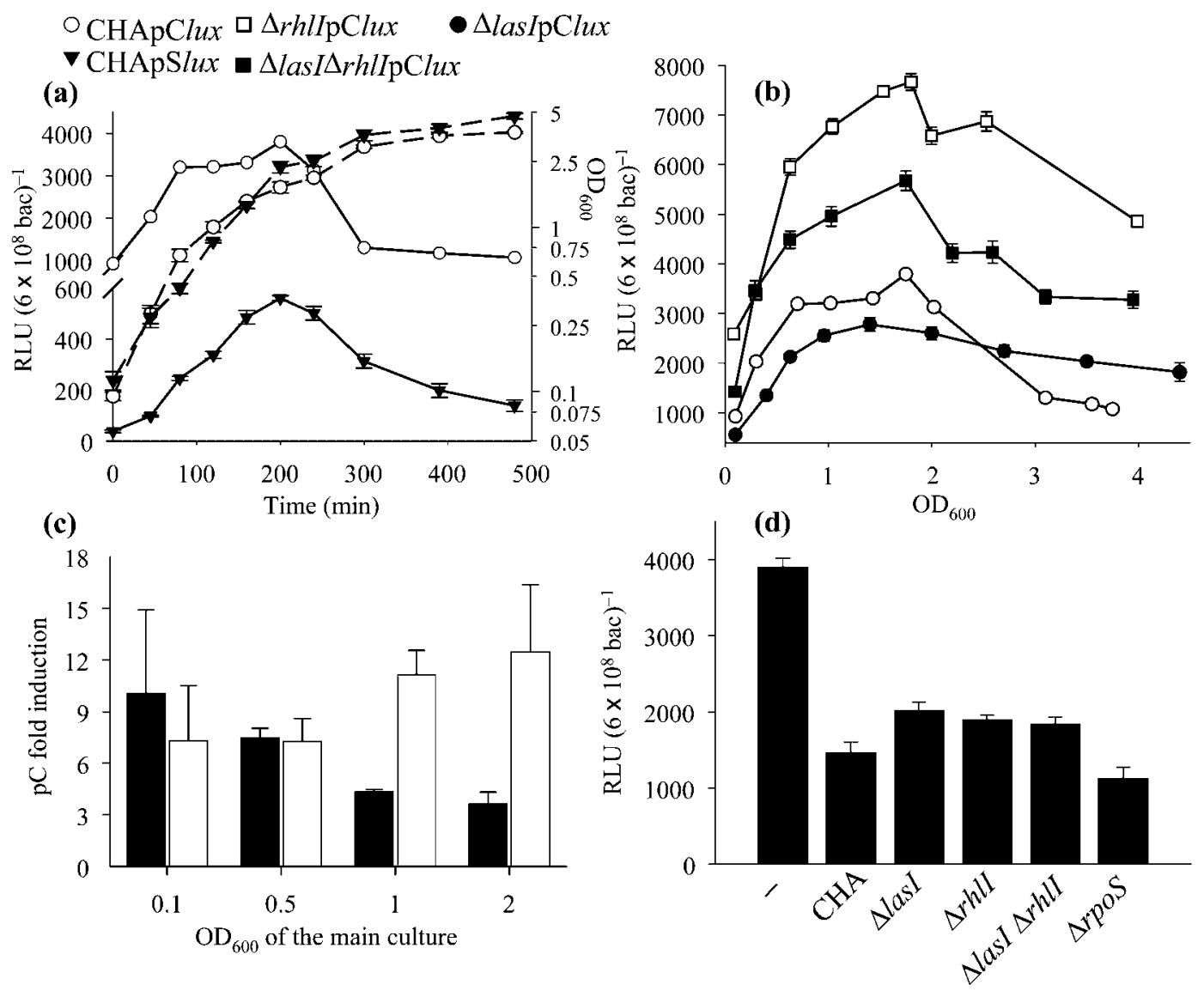

Fig. 1. Stationary-phase culture supernatants inhibit T3SS expression. (a, b) T3SS expression is dependent on cell density. (a) Wild-type CHA carrying a single copy of $\mathrm{pC}:$ : lux or a pS : : lux transcriptional reporter on its chromosome was grown at $37{ }^{\circ} \mathrm{C}$ in LB under T3SS-inducing conditions. At different times during bacterial growth, $\mathrm{pC}$ and pS promoter activity was measured by luminescence (RLU) and normalized to bacterial counts $\left(6 \times 10^{8}\right.$ bacteria; bac) (continuous lines, left axis). OD 600 values of cultures at the selected time points are represented by dashed lines (right axis). Results represent the mean \pm SE of triplicate samples, and are based on at least three independent experiments. $\bigcirc, \mathrm{CHApClux} ; \boldsymbol{\nabla}, \mathrm{CHApS} / u x$. (b) pC activity during growth of the wild-type $\mathrm{CHA}$ or its isogenic mutants ( $\Delta /$ asl, $\Delta r h / l$ and $\Delta / a s / \Delta r h / l)$ carrying a single copy of $\mathrm{pC}:$ : /ux, was measured by luminescence (RLU) and normalized to bacterial count $\left(6 \times 10^{8}\right.$ bacteria; bac). $\bigcirc$, CHApClux; $\square, \Delta$ rh/lpClux; $\mathbf{\square}, \Delta / a s /-$ rhl/pClux; $-\Delta$ las/pClux. (c) Fold-induction of T3SS from a culture of CHA (plApCgfp) (main culture) at different growth stages, represented by $\mathrm{OD}_{600}$. Before T3SS induction, the $\mathrm{OD}_{600}$ of the main culture was kept constant (black bars) or set to 0.1 (white bars), and the medium was replaced with fresh medium. Results represent $\mathrm{pC}$ fold-induction. Bars are the mean $\pm \mathrm{SE}$ of three independent experiments. (d) Stationary-phase culture supernatants inhibit exSCEBA expression independent of QS signals. The wild-type $\mathrm{CHA}\left(\mathrm{pC}:: / \mathrm{lu}\right.$ ) was grown at $37^{\circ} \mathrm{C}$ in $\mathrm{LB}$ under T3SS-inducing conditions, supplemented or not supplemented with concentrated stationary-phase supernatants from CHA, $\Delta / a s l, \Delta r h l l, \Delta / a s /-r h / l$ or $\Delta r p o S$, as indicated. -, No supernatant, but VB added. The final relative concentration of supernatant was $1 \times$. RLU were measured at $\mathrm{OD}_{600} \sim 1.7$ and normalized to bacterial counts $\left(6 \times 10^{8}\right.$ bacteria; bac). Bars represent the mean \pm SE of triplicate samples and are based on three independent experiments.

(Fletcher et al., 2007). Stationary-phase culture supernatants from these strains were produced as indicated in Methods and tested for T3SS repression activity. Cultures of CHA ( $\mathrm{pC}:: \operatorname{lux}$ ) with the PAO1 or the PQS-productionmutant supernatant had RLU levels (RLU/OD 600 ) of $247.8 \pm 16.08$ and $215.2 \pm 21.9$, respectively, compared with $1678.7 \pm 326.5$ without supernatant. Thus, two P. aeruginosa strains other than CHA, PAO1 and a PAO1 mutant unable to produce PQS, are also able to secrete the T3SS inhibitory molecule(s). These results indicate the following: (i) there is/are T3SS-inhibiting signal(s) in the stationaryphase culture supernatants; (ii) known $P$. aeruginosa QS molecules (3-oxo-C12-HSL, C4-HSL and PQS) are not the inhibitory signal; and (iii) AHL synthases LasI, RhlI and the AHQ synthesis pathway, which starts with PqsA, are not implicated in the production of such T3SS-inhibiting signal(s). Considering the important role of RpoS, a recognized stationary-phase central regulator (Loewen 
Trp catabolite signals in $P$. aeruginosa T3SS expression

Table 3. QS signal production in different $P$. aeruginosa strains

Values are in pmol.

\begin{tabular}{|lrc|}
\hline Strain & C4-HSL & 3-oxo-C12-HSL \\
\hline CHA & 8399 & 29.8 \\
DlasI & 4149 & 0 \\
DrhlI & 0 & 1.6 \\
DlasIrhlI & 0 & 0 \\
\hline
\end{tabular}

et al., 1998), which was recently identified as an inhibitor of T3SS expression (Hogardt et al., 2004; Shen et al., 2006), we prepared the stationary-phase culture supernatant from a $\triangle r p o S$ mutant and tested its role in exsCEBA expression. As shown in Fig. 1(d), deletion of rpoS does not change the inhibitory role of the supernatant on exsCEBA expression. Taken together, these results suggest the presence of unknown T3SS-inhibiting signals in the stationary-phase culture supernatants that are distinct from known QS signals, and whose synthesis is not dependent on QS signal synthesis.

\section{Isolation and characterization of mutants impaired in T3SS-inhibiting signal(s) production}

To identify the gene(s) involved in the production of the T3SS-inhibiting signal(s) in the stationary-phase culture supernatants, a library of $\sim 10^{6} \mathrm{Gm}$-resistant mutants was generated with the mariner-based transposon Himarl:: $\mathrm{Gm}^{\mathrm{r}}$ using plasmid pFAC (Diaz-Perez et al., 2004). Roughly 2000 mutants were screened in 96-well plates for the effect of their stationary-phase culture supernatants on the expression of exoS. Twenty mutants whose supernatants seemed to lose their ability to inhibit exoS expression were selected in the first round of screening. After a second round of close observation, two mutants with a confirmed phenotype were further analysed by cloning their PstI-digested genomic DNA fragments (flanked by the transposon) into the plasmid pUC18 and then sequencing. A Southern blot analysis revealed that the mutation was caused by a single transposition event (data not shown). Sequence comparison against the PAO1 genome (http://www.pseudomonas.com; Stover et al., 2000) showed that the first transposon insertion occurred inside the ORF of $\operatorname{trpA}$ (PA0035), which encodes the Trp synthase chain involved in the biosynthesis of Trp (Hadero \& Crawford, 1986), and the second occurred in leuC (PA3121), which encodes the 3-isopropylmalate dehydratase large subunit involved in the biosynthesis of leucine and isoleucine. The $\operatorname{trp} A$ mutant was selected for further investigations in this study because the affected gene is involved in the biosynthesis of Trp, a precursor of several important signalling molecules in plants and bacteria, including IAA (also termed auxin in plants), kynurenine and PQS (Fig. 2, Supplementary Table S1). Furthermore, $\mathrm{PAO}$ genome analysis revealed that all the genes required for the synthesis of these signalling molecules exist in the genome of $P$. aeruginosa (Fig. 2, Supplementary Table S2).

\section{Trp is involved in the production of T3SS- inhibiting signal(s)}

To determine the role of $\operatorname{trp} A$ in the production of T3SSinhibiting signal(s) during the stationary phase, $\Delta \operatorname{trp} A$, a mutant strain with an unmarked in-frame deletion of $\operatorname{trp} A$, was constructed through allelic exchange as described in Methods and confirmed by PCR (data not shown). Stationary-phase culture supernatants from this mutant were added to CHA ( $\mathrm{pC}:: \operatorname{lu} x)$ cultured from an initial $\mathrm{OD}_{600}$ of 0.05 in LB under T3SS-inducing conditions. In contrast to the stationary-phase culture supernatants from the wild-type CHA, supernatants from $\triangle \operatorname{trpA}$ lost their ability to inhibit exsCEBA expression and gave the same profile as a control in which supernatant was replaced by $\mathrm{VB}$, the minimal medium used for the preparation of supernatants (Fig. 3). The addition of $200 \mu \mathrm{g} \operatorname{Trp~ml}^{-1}$ in $\mathrm{VB}$ to the $\triangle \operatorname{trp} A$ mutant culture restored the production of T3SS-inhibiting signal(s) from $\Delta \operatorname{trp} A$. However, the addition of Trp during the preparation of supernatants from wild-type CHA did not change the ability of the supernatants to inhibit exsCEBA expression compared with the non-Trp-amended supernatants (Fig. 3). These results suggest that Trp might be involved in the production of T3SS-inhibiting signal(s) during the stationary phase, but that Trp itself is not the signal.

\section{IAA, NAA and 3-hydroxykynurenine inhibit exsCEBA expression}

Trp can serve as the precursor of IAA (Cohen et al., 2003), the first plant hormone discovered, which is also termed auxin (Woodward \& Bartel, 2005). It was therefore necessary to ascertain whether or not Trp and Trpderivatives play a direct role in the regulation of T3SS expression. Different concentrations of Trp (up to $2.5 \mathrm{mM}$ ) were added to the CHA (pC::lux), cultured from an initial $\mathrm{OD}_{600}$ of 0.05 in $\mathrm{LB}$ under T3SS-inducing and non-inducing conditions, but no transcriptional differences were observed (data not shown). However, as shown in Fig. 4(a), $1 \mathrm{mM}$ IAA had an evident inhibitory effect on exsCEBA expression but did not inhibit bacterial growth. We also tested several commercial auxin-like compounds, including IBA, NAA and 2,4-D (Woodward \& Bartel, 2005), but only NAA inhibited exsCEBA expression (Fig. 4a). Also, $1 \mathrm{mM}$ 3-hydroxykynurenine, but not kynurenine, inhibited exsCEBA expression (Fig. 4a). Furthermore, no transcriptional difference was observed in the presence of up to $20 \mu \mathrm{M}$ PQS (data not shown), excluding a role for this QS signal in the T3SS inhibition described. To test the catabolic pathway derived from tryptophan that leads to kynurenin (Fig. 2), we constructed a kynA mutant and tested the inhibitory effect of a stationary-phase supernatant from this strain on T3SS activation. Unlike the $\Delta \operatorname{trp} A$ strain, which no longer 


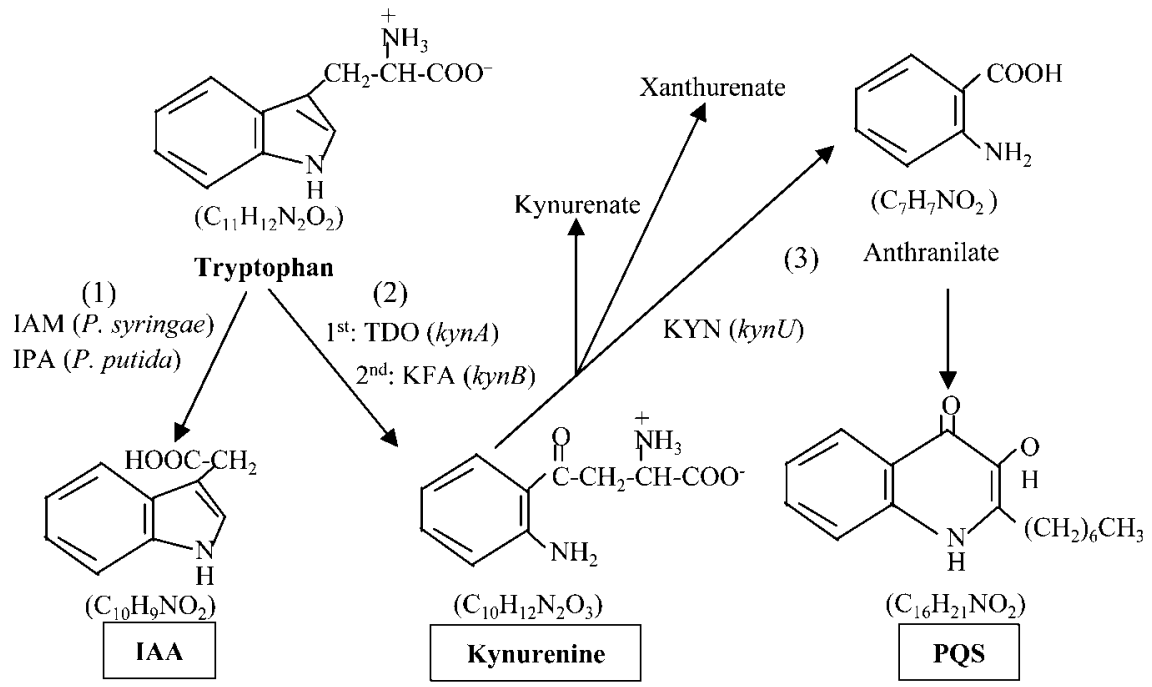

Fig. 2. Trp is a precursor of several important signalling molecules in Pseudomonas spp. (1) Trp-dependent IAA biosynthesis. The two most common routes for IAA biosynthesis in bacteria are the indole-3-acetamide (IAM) pathway and indole-3-pyruvic acid (IPA) pathway (Lambrecht et al., 2000). In Pseudomonas syringae, Trp monooxygenase (laaM) converts Trp to IAM, and an IAM hydrolase (laaH) converts IAM to IAA (Patten \& Glick, 1996). Ipdc, the gene that encodes indolepyruvate decarboxylase and the key gene in the IPA pathway for IAA synthesis, has been isolated from Pseudomonas putida (Patten \& Glick, 2002). (2) Degradation of Trp to kynurenine. In P. aeruginosa, tryptophan 2,3-dioxygenase (TDO), encoded by kynA (PA2579), and kynurenine formamidase (KFA), encoded by kynB (PA 2081), are involved in this pathway (Kurnasov et al., 2003). (3) PQS biosynthesis. Anthranilate is the precursor of PQS (Calfee et al., 2001), and all enzymes required in the anthranilate pathway via Trp from P. aeruginosa have been characterized (Kurnasov et al., 2003). KYN, kynureninase, encoded by kynU (PA2080).

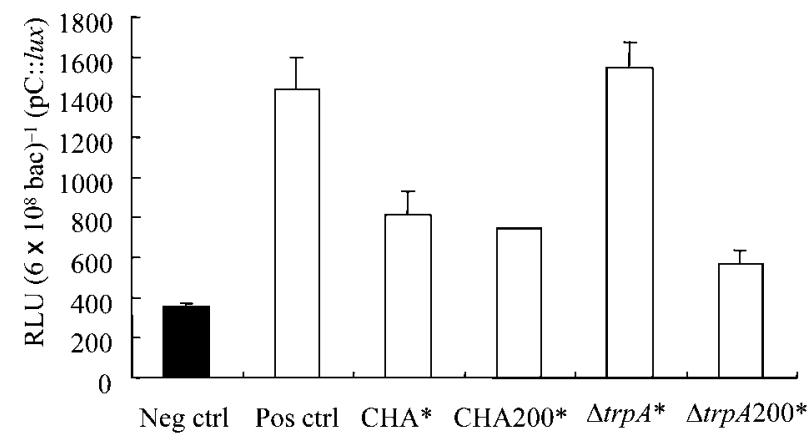

Fig. 3. Trp is implicated in the production of T3SS-inhibiting signal(s). Wild-type CHA carrying a single copy of the $\mathrm{pC}:$ : lux transcriptional reporter on its chromosome was grown at $37^{\circ} \mathrm{C}$ in LB in the presence of the stationary-phase culture supernatants (indicated by asterisks). These were prepared in VB (CHA and $\Delta \operatorname{trp} A)$ or VB supplemented with $200 \mu \mathrm{g} \mathrm{Trp} \mathrm{ml}^{-1}$ (CHA200 and $\Delta \operatorname{trp} A 200)$. RLU were measured over $4 \mathrm{~h}$ of culture and normalized to bacterial counts $\left(6 \times 10^{8}\right.$ bacteria; bac). Bars represent the mean $\pm \mathrm{SE}$ of triplicate samples and are derived from three independent experiments. White and black bars represent T3SSinducing and non-inducing conditions, respectively. Neg ctrl and Pos ctrl are negative and positive induction controls, respectively, in which VB (instead of supernatants) was added. produces a T3SS-inhibiting signal (Fig. 3), the kynA mutant still produced some T3SS-inhibiting signal, but much less than the wild-type. Indeed, CHA (pC::lux) grown in the presence of the $k y n A$ mutant supernatant showed an RLU level, representing T3SS activation, of $689.7 \pm 23.7 \mathrm{RLU} / \mathrm{OD}_{600}$. Culture grown without supernatant had an RLU level of $959.7 \pm 68 \mathrm{RLU} \mathrm{OD}_{600}$, and the CHA supernatant induced a stronger repression $\left(121.8 \pm 4.7 \mathrm{RLU} / \mathrm{OD}_{600}\right)$. This result suggests that both the kynurenine-derived molecules and the IAA-derived molecules, both of which are Trp-derived molecules, could play a role in the downregulation of T3SS expression. This is indirectly supported by the fact that 3-hydroxykynurenine partially inhibited T3SS expression (Fig. 4a).

In addition to calcium depletion, in vitro growth in the presence of serum also leads to generalized T3SS expression and secretion (Vallis et al., 1999). Therefore, we asked whether auxin compounds could inhibit the T3SS expression induced by human serum. As shown in Fig. 4(b), exs $C E B A$ transcription was partially inhibited in the presence of $1 \mathrm{mM}$ IAA or NAA, but not with 2,4-D or IBA. No $\mathrm{pH}$ change or growth difference was observed to arise from the use of either IAA or IAA-like compounds. Considering the fact that IBA is completely identical to IAA except for two additional methylene groups in the side chain, but has no effect on the expression of the T3SS induced by either calcium depletion or serum, we 
(a)
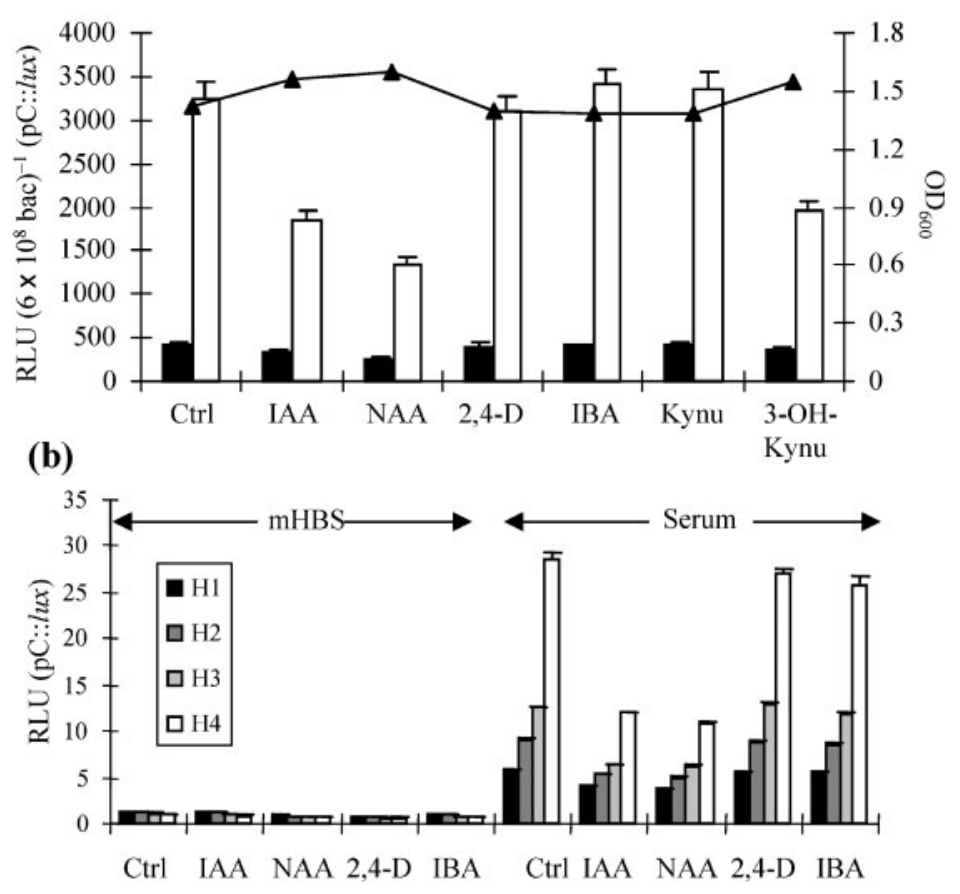

Fig. 4. Trp derivatives inhibit T3SS expression. (a) IAA, NAA and 3-hydroxykynurenine inhibit EGTA-induced exsCEBA expression. Wild-type CHA carrying a single copy of the $\mathrm{pC}$ : : lux transcriptional reporter in its chromosome was grown at $37^{\circ} \mathrm{C}$ in $\mathrm{LB}$ in the absence or presence of $1 \mathrm{mM}$ of the indicated commercial molecules under T3SS-inducing (white bars) or non-inducing conditions (black bars), as described in Methods. RLU were measured after $4 \mathrm{~h}$ of culture and related to normalized bacterial counts $\left(6 \times 10^{8}\right.$ bacteria; bac). Bars represent the mean \pm SE of triplicate samples and are derived from three independent experiments. $\boldsymbol{\Delta}, \mathrm{OD}_{600}$ under T3SSinducing conditions. Kynu, kynurenine; Ctrl, control condition in which no molecules were added. (b) IAA and NAA inhibit serum-induced exsCEBA expression. A culture of the wildtype $\mathrm{CHA}$ strain $\left(5 \times 10^{5}\right.$ bacteria) carrying a single copy of the $\mathrm{pC}:$ :lux transcriptional reporter on its chromosome was incubated with $\mathrm{mHBS}$ buffer or $10 \%$ human serum in the absence or presence of $1 \mathrm{mM}$ of the indicated molecules in a 96-well plate. RLU were measured each hour over $4 \mathrm{~h}$ of incubation $(\mathrm{H} 1$ to $\mathrm{H} 4)$. Bars represent the mean \pm SE of triplicate samples and are derived from two independent experiments. Ctrl, control condition in which no molecules were added. concluded that IAA and NAA could inhibit exsCEBA expression specifically.

\section{IAA and NAA inhibit type III effector secretion expression downstream of ExsA}

The secretion of T3SS effectors strongly correlates with $P$. aeruginosa virulence both in animal models and in studies of human disease (Roy-Burman et al., 2001). To examine whether IAA and NAA are involved in the inhibition of type III effector secretion, culture supernatants were analysed from wild-type CHA containing plasmid pexsAind, which can express exsA in trans via induction by IPTG. When the expression of the exoS transcriptional activator is induced using this construct, it allows T3SS gene expression and toxin production even in the presence of calcium (Epaulard et al., 2006; Filopon et al., 2006). As shown in Fig. 5, the growth of bacteria in the presence of EGTA resulted in type III effector secretion. However such secretion was prevented when $1 \mathrm{mM}$ NAA or IAA (data not shown) was added, even when exsA was expressed in trans at the same time. To distinguish between inhibition of type III secretory activity or inhibition of ExsAdependent activation, we measured the intrabacterial level of the ExoS and ExoT effectors with an anti-ExoS/T serum.
Fig. 5(b) indicates that even in the presence of in transexpressed exsA, the levels of both ExoS and ExoT were dramatically diminished when NAA was added. This result suggests that IAA and NAA could negatively regulate type III effector expression by blocking the activation downstream of ExsA.

\section{DISCUSSION}

$P$. aeruginosa T3SS expression is controlled by multicomponent regulatory networks, which integrate a diverse set of environmental cues and whose most likely function is to restrict the energy-consuming expression of 43 genes to the correct place and time (Yahr \& Wolfgang, 2006). It would not be surprising if a mechanism exists to arrest T3SS gene expression when not required, since a mechanism exists to activate all of the T3SS operons when necessary through the activation of the ExsA-mediated positive regulatory loop. In this study we showed that the stationary-phase culture supernatants of at least two $P$. aeruginosa strains significantly repress T3SS gene expression (by $\sim 60 \%$ with the tested concentration), and we described our attempts to discover the nature of such T3SS-inhibiting signal(s). 


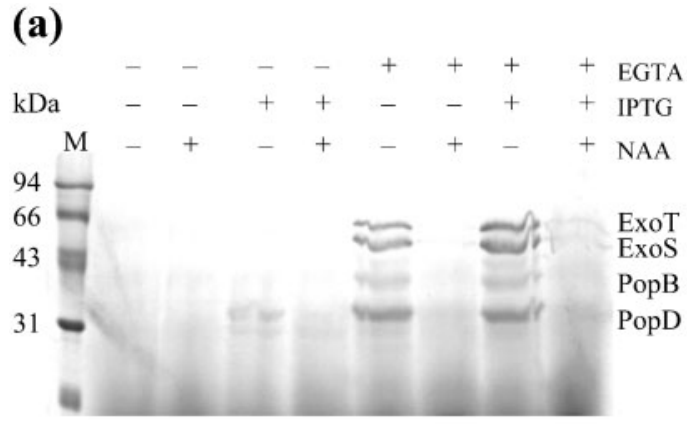

(b)

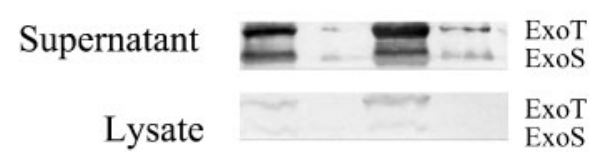

Fig. 5. NAA inhibits T3SS expression even in the presence of in trans-expressed ExsA. (a) Wild-type CHA expressing exogenous ExsA from IPTG-inducible plasmid pexsAind was grown in the absence (-) or presence (+) of 1 mM NAA under T3SS-inducing $(E G T A+)$ or non-inducing conditions (EGTA-). IPTG was used at $1 \mathrm{mM}$ concentration. Supernatants were normalized to bacterial counts $\left(6 \times 10^{8}\right.$ per lane) and subjected to TCA precipitation. The extracellular proteins were separated by $10 \%$ SDS-PAGE and stained with Coomassie Blue. $\mathrm{M}$, protein marker; sizes in $\mathrm{kDa}$ are indicated on the left. (b) Western blot of normalized supernatants or bacterial lysates after separation by $10 \%$ SDS-PAGE, transfer onto nitrocellulose membranes and revelation with an anti-ExoS/T serum at $1: 1000$ dilution.

Considering that QS is a generic regulatory mechanism that controls the expression of virulence genes, including T3SS genes, in a population-density-dependent manner (Juhas et al., 2005), we first ruled out the possibility that such strong T3SS-inhibiting signal(s) are associated with the QS system (Fig. 1d) and showed that neither acyl-HSLs nor PQS are involved in T3SS repression. Transposition mutants allowed us to find evidence that the production of the T3SS-inhibiting signal(s) is Trp-dependent (Fig. 3). Thus tryptophan catabolites accumulate in culture supernatant during growth and act as a QS signal to inhibit T3SS gene expression. In the experiment described in Fig. 1(c), cells from a stationary-phase culture were sedimented, suspended in fresh growth medium and assayed for T3SS gene expression. One would presume that suspending the cells in fresh growth medium would dilute the inhibitory catabolites, and that T3SS gene expression would be restored, contradicting our results. Nevertheless, production of such a signal should not be shut off immediately after medium replacement and should again rapidly reach inhibitory levels in high-cell-density culture due both to the high number of bacteria secreting the catabolites and to a possible auto-induction mechanism to induce their production, similar to the classic QS signalling system. Considering the fact that tryptophan is the precursor of several important signalling molecules, including IAA and kynurenine, we tested these commercially available molecules in order to investigate their roles in the regulation of T3SS expression.

IAA is critical for plant growth and development, and is often effective at sub-micromolar concentrations in plants (Woodward \& Bartel, 2005). Although it is widely accepted that plants use both Trp-dependent and Trp-independent routes to synthesize IAA, the relevant genes, enzymes and intermediates of these pathways have not yet all been identified. Many bacteria are also able to produce IAA, mainly by using a Trp-dependent pathway (Lambrecht et al., 2000), and such a pathway is indeed observed in Pseudomonas spp. (Patten \& Glick, 2002) (Fig. 2). Recent studies have shown that several strains of $P$. aeruginosa isolated from soil (Bano \& Musarrat, 2003) can produce IAA, and a maximum production of IAA of $19.1 \mathrm{mg} \mathrm{ml}^{-1}$ (equivalent to $0.1 \mathrm{mM}$ ) by strain $P$. aeruginosa FP10 has been observed after $42 \mathrm{~h}$ incubation in Dworkin and Foster (DF) salts medium supplemented with $500 \mu \mathrm{g} \operatorname{Trp~} \mathrm{ml}^{-1}$ (Ayyadurai et al., 2006).

IAA and a compound similar to IAA, NAA, can indeed inhibit T3SS expression and type III effector secretion at millimolar concentrations (Figs 4 and 5). Although one study has recently shown that IAA can inhibit the expression of genes required for the transfer of T-DNA (type IV secretion) in Agrobacterium tumefaciens via VirA, a sensor kinase of a two-component regulatory system (Liu \& Nester, 2006), we found that P. aeruginosa PA1243 (www.pseudomonas.com) (Stover et al., 2000), which has $49 \%$ similarity to VirA, was not involved in the IAAdependent inhibition of T3SS expression (data not shown). Since the transcription of the T3SS is coupled with secretion (Urbanowski et al., 2005; Rietsch et al., 2005), it is possible that IAA or NAA blocks the secretion channel and thus blocks the activation of ExsA and the transcriptional activation of T3SS genes. Indeed, an equivalent process has been demonstrated for recently identified inhibitory molecules that act as 'calcium mimetics' for Yersinia type III secretion (Nordfelth et al., 2005). However, in our case we showed clearly that NAA is able to inhibit effector production even in the presence of in trans-expressed exs $A$, suggesting that the signal transduction pathway that responds to tryptophan derivatives acts downstream of exsA transcription activation of T3SS genes. Recently, one study has shown that RetS/LadS/GacAS, twocomponent regulatory systems in $P$. aeruginosa, are implicated in the transition between activation of the T3SS (acute infection) and biofilm (chronic infection) via a post-transcriptional regulation of targeted genes after the detection of an unknown signal (Ventre et al., 2006). Therefore, it would be interesting to investigate whether the signals described here are the same as those detected by the RetS/LadS/GacAS pathway.

IAA itself was not detected in our stationary-phase culture supernatants using Salkowski's reagent (Patten \& Glick, 2002). Thus, IAA does not seem to be the natural 
T3SS-inhibiting signal, although genes encoding monooxygenase and aminotransferase, enzymes needed in the Trpdependent biosynthesis of IAA (Supplementary Table S2), do exist in the genome of $P$. aeruginosa PAO1 (www.pseudomonas.com) (Stover et al., 2000). However, we still have evidence that T3SS-inhibiting signals could be Trp catabolites, such as NAA and 3-hydroxykynurenine, because (i) these compounds may be Trp-derived, (ii) they have an inhibitory effect on T3SS expression, and (iii) a kynA mutant supernatant loses some ability to induce repression of T3SS genes. Since there are at least seven potential genes involved in the production of IAA (Supplementary Table S2), we have not examined all of their roles; however, a thorough examination of these genes would provide a more precise profile of the role of tryptophan catabolites in the regulation of T3SS expression.

The reason why Trp-derived catabolites, such as IAA, NAA and kynurenin-derived molecules, are directly or indirectly involved in the regulation of T3SS expression remains to be discovered, but we do know that metabolic signals/stresses have a profound influence on T3SS expression. Mutants that lack pyruvate dehydrogenase (aceA or aceB) (Dacheux et al., 2002) or a glucose transport regulator $(g l t R)$ (Wolfgang et al., 2003) fail to induce T3SS expression under calciumdepletion conditions. Similarly, overproduction of multidrug (MDR) efflux pumps (Linares et al., 2005) or overexpression of genes involved in histidine transport and metabolism (Rietsch et al., 2004) prevents T3SS expression. In our study, we have not further examined the leuC transposon mutant because its phenotype is less apparent than that of $\triangle \operatorname{trp} A$. However, the supernatant of the leuC transposon mutant did lose some ability to inhibit T3SS expression, further suggesting that metabolic signals/stresses play an important role in the regulation of T3SS expression, or that multiple pathways act synergistically to prevent T3SS activation at high cell density.

A recent study has suggested that one catabolite controlling T3SS expression is derived from acetyl-CoA (Rietsch \& Mekalanos, 2006); another study has shown that IAA $(0.5 \mathrm{mM})$ is capable of inducing changes in gene expression, enzyme activity and metabolite level in central metabolic pathways in E. coli, with a 2.7-fold increase in the production of acetyl-CoA (Bianco et al., 2006). It follows that the relationship between IAA, acetyl-CoA and T3SS expression needs further investigation. Finally, it appears that a combination of different metabolic or stress signals, either secreted (such as Trp catabolites) or acting directly inside the bacterial cells, is involved in the downregulation of T3SS.

We are convinced that targeting virulence gene expression represents a new method for antibacterial treatment, as shown recently for Vibrio cholerae, whereby a smallmolecule inhibitor of both toxin and pili expression was shown to protect infant mice from colonization (Hung et al., 2005). Two different small-molecule inhibitors of T3SS have also been identified as potential drugs in the treatment of Chlamydia trachomatis, a Gram-negative bacterium causing sexually transmitted disease and preventable blindness worldwide (Wolf et al., 2006). Thus, the role of Trp catabolites in the acute infection of $P$. aeruginosa and of their receptors on the surface of bacteria remains an interesting area of study. Very recently, another Trp oxidative metabolite, 3-hydroxyoxindole, has been reported to be involved in the regulation of the virulence of Ralstonia solanacearum (Delaspre et al., 2007).

Finally, it has also been proven that auxin is the signal for plant-microbe interactions in symbiosis. The question therefore arises concerning the beneficial effects of auxinlike molecules on $P$. aeruginosa during chronic colonization. Interestingly, the signal seen in this study, which is used by $P$. aeruginosa to stop T3SS activity, could at the same time allow the bacteria to become toxic to neutrophils or macrophages, since IAA-like compounds have been shown to be toxic to these cells (Pires de Melo et al., 1998). Furthermore, it has been shown that tryptophan metabolites such as kynurenin, 3-hydroxykynurenin and anthranilic acids suppress the $\mathrm{T}$-cell response in humans (Terness et al., 2002). These compounds act synergistically to induce T-cell cytotoxicity. It has also been demonstrated recently that QS molecules can act as immunomodulators (Pritchard, 2006). It therefore appears essential to investigate the influence of these novel signalling molecules derived from tryptophan in the modulation of host physiological functions and hence in the virulence of $P$. aeruginosa.

\section{ACKNOWLEDGEMENTS}

We thank J. Campos-Garcia, Universidad Michoacana de San Nicolás de Hidalgo, Michoacán, Mexico, for providing plasmid pFAC, P. Williams, University of Nottingham, UK, for providing strains PAO1 and PAO1 pqsA, and also D. Haras for the quantification of HSL in supernatants. We also thank Gareth Butt for correcting the final draft. S.D.K. was supported by a $\mathrm{PhD}$ scholarship from the Agence Universitaire de la Francophonie.

\section{REFERENCES}

Ayyadurai, N., Ravindra Naik, P., Sreehari Rao, M., Sunish Kumar, R., Samrat, S. K., Manohar, M. \& Sakthivel, N. (2006). Isolation and characterization of a novel banana rhizosphere bacterium as fungal antagonist and microbial adjuvant in micropropagation of banana. $J$ Appl Microbiol 100, 926-937.

Bano, N. \& Musarrat, J. (2003). Characterization of a new Pseudomonas aeruginosa strain $\mathrm{NJ}-15$ as a potential biocontrol agent. Curr Microbiol 46, 324-328.

Bianco, C., Imperlini, E., Calogero, R., Senatore, B., Pucci, P. \& Defez, R. (2006). Indole-3-acetic acid regulates the central metabolic pathways in Escherichia coli. Microbiology 152, 2421-2431.

Bleves, S., Soscia, C., Nogueira-Orlandi, P., Lazdunski, A. \& Filloux, A. (2005). Quorum sensing negatively controls type III secretion regulon expression in Pseudomonas aeruginosa PAO1. J Bacteriol 187, 3898-3902. 
Bodey, G. P., Bolivar, R., Fainstein, V. \& Jadeja, L. (1983). Infections caused by Pseudomonas aeruginosa. Rev Infect Dis 5, 279-313.

Calfee, M. W., Coleman, J. P. \& Pesci, E. C. (2001). Interference with Pseudomonas quinolone signal synthesis inhibits virulence factor expression by Pseudomonas aeruginosa. Proc Natl Acad Sci U S A 98, 11633-11637.

Cohen, J. D., Slovin, J. P. \& Hendrickson, A. M. (2003). Two genetically discrete pathways convert tryptophan to auxin: more redundancy in auxin biosynthesis. Trends Plant Sci 8, 197-199.

Dacheux, D., Attree, I., Schneider, C. \& Toussaint, B. (1999). Cell death of human polymorphonuclear neutrophils induced by a Pseudomonas aeruginosa cystic fibrosis isolate requires a functional type III secretion system. Infect Immun 67, 6164-6167.

Dacheux, D., Toussaint, B., Richard, M., Brochier, G., Croize, J. \& Attree, I. (2000). Pseudomonas aeruginosa cystic fibrosis isolates induce rapid, type III secretion-dependent, but ExoU-independent, oncosis of macrophages and polymorphonuclear neutrophils. Infect Immun 68, 2916-2924.

Dacheux, D., Attree, I. \& Toussaint, B. (2001). Expression of ExsA in trans confers type III secretion system-dependent cytotoxicity on noncytotoxic Pseudomonas aeruginosa cystic fibrosis isolates. Infect Immun 69, 538-542.

Dacheux, D., Epaulard, O., de Groot, A., Guery, B., Leberre, R., Attree, I., Polack, B. \& Toussaint, B. (2002). Activation of the Pseudomonas aeruginosa type III secretion system requires an intact pyruvate dehydrogenase aceAB operon. Infect Immun 70, 3973-3977.

Dahan, S., Knutton, S., Shaw, R. K., Crepin, V. F., Dougan, G. \& Frankel, G. (2004). Transcriptome of enterohemorrhagic Escherichia coli $\mathrm{O} 157$ adhering to eukaryotic plasma membranes. Infect Immun 72, 5452-5459.

Dasgupta, N., Lykken, G. L., Wolfgang, M. C. \& Yahr, T. L. (2004). A novel anti-anti-activator mechanism regulates expression of the Pseudomonas aeruginosa type III secretion system. Mol Microbiol 53, 297-308.

Delaspre, F., Nieto Penalver, C. G., Saurel, O., Kiefer, P., Gras, E., Milon, A., Boucher, C., Genin, S. \& Vorholt, J. A. (2007). The Ralstonia solanacearum pathogenicity regulator $\operatorname{HrpB}$ induces 3-hydroxyoxindole synthesis. Proc Natl Acad Sci U S A 104, 15870-15875.

Delic-Attree, I., Toussaint, B. \& Vignais, P. M. (1995). Cloning and sequence analyses of the genes coding for the integration host factor (IHF) and HU proteins of Pseudomonas aeruginosa. Gene 154, 61-64.

Della Chiesa, M., Carlomagno, S., Frumento, G., Balsamo, M., Cantoni, C., Conte, R., Moretta, L., Moretta, A. \& Vitale, M. (2006). The tryptophan catabolite L-kynurenine inhibits the surface expression of NKp46- and NKG2D-activating receptors and regulates NK-cell function. Blood 108, 4118-4125.

Diaz-Perez, A. L., Zavala-Hernandez, A. N., Cervantes, C. \& Campos-Garcia, J. (2004). The gnyRDBHAL cluster is involved in acyclic isoprenoid degradation in Pseudomonas aeruginosa. Appl Environ Microbiol 70, 5102-5110.

Epaulard, O., Toussaint, B., Quenee, L., Derouazi, M., Bosco, N., Villiers, C., Le Berre, R., Guery, B., Filopon, D. \& other authors (2006). Anti-tumor immunotherapy via antigen delivery from a live attenuated genetically engineered Pseudomonas aeruginosa type III secretion system-based vector. Mol Ther 14, 656-661.

Eriksson, S., Lucchini, S., Thompson, A., Rhen, M. \& Hinton, J. C. (2003). Unravelling the biology of macrophage infection by gene expression profiling of intracellular Salmonella enterica. Mol Microbiol 47, 103-118.

Faucher, S. P., Porwollik, S., Dozois, C. M., McClelland, M. \& Daigle, F. (2006). Transcriptome of Salmonella enterica serovar Typhi within macrophages revealed through the selective capture of transcribed sequences. Proc Natl Acad Sci U S A 103, 1906-1911.

Feltman, H., Schulert, G., Khan, S., Jain, M., Peterson, L. \& Hauser, A. R. (2001). Prevalence of type III secretion genes in clinical and environmental isolates of Pseudomonas aeruginosa. Microbiology 147, 2659-2669.

Filopon, D., Merieau, A., Bernot, G., Comet, J. P., Leberre, R., Guery, B., Polack, B. \& Guespin-Michel, J. (2006). Epigenetic acquisition of inducibility of type III cytotoxicity in $P$. aeruginosa. BMC Bioinformatics 7, 272.

Fletcher, M. P., Diggle, S. P., Cámara, M. \& Williams, P. (2007). Biosensor-based assays for PQS, HHQ and related 2-alkyl-4quinolone quorum sensing signal molecules. Nat Protoc 2, 1254-1262.

Frumento, G., Rotondo, R., Tonetti, M., Damonte, G., Benatti, U. \& Ferrara, G. B. (2002). Tryptophan-derived catabolites are responsible for inhibition of $\mathrm{T}$ and natural killer cell proliferation induced by indoleamine 2,3-dioxygenase. J Exp Med 196, 459-468.

Fuqua, W. C., Winans, S. C. \& Greenberg, E. P. (1994). Quorum sensing in bacteria: the LuxR-LuxI family of cell density-responsive transcriptional regulators. J Bacteriol 176, 269-275.

Garau, J. \& Gomez, L. (2003). Pseudomonas aeruginosa pneumonia. Curr Opin Infect Dis 16, 135-143.

Goodman, A. L., Kulasekara, B., Rietsch, A., Boyd, D., Smith, R. S. \& Lory, S. (2004). A signaling network reciprocally regulates genes associated with acute infection and chronic persistence in Pseudomonas aeruginosa. Dev Cell 7, 745-754.

Ha, U. \& Jin, S. (2001). Growth phase-dependent invasion of Pseudomonas aeruginosa and its survival within HeLa cells. Infect Immun 69, 4398-4406.

Ha, U. H., Kim, J., Badrane, H., Jia, J., Baker, H. V., Wu, D. \& Jin, S. (2004). An in vivo inducible gene of Pseudomonas aeruginosa encodes an anti-ExsA to suppress the type III secretion system. Mol Microbiol 54, 307-320.

Hadero, A. \& Crawford, I. P. (1986). Nucleotide sequence of the genes for tryptophan synthase in Pseudomonas aeruginosa. Mol Biol Evol 3, 191-204.

Hauser, A. R., Cobb, E., Bodi, M., Mariscal, D., Valles, J., Engel, J. N. \& Rello, J. (2002). Type III protein secretion is associated with poor clinical outcomes in patients with ventilator-associated pneumonia caused by Pseudomonas aeruginosa. Crit Care Med 30, 521-528.

Hoang, T. T., Karkhoff-Schweizer, R. R., Kutchma, A. J. \& Schweizer, H. P. (1998). A broad-host-range Flp-FRT recombination system for site-specific excision of chromosomally-located DNA sequences: application for isolation of unmarked Pseudomonas aeruginosa mutants. Gene 212, 77-86.

Hogardt, M., Roeder, M., Schreff, A. M., Eberl, L. \& Heesemann, J. (2004). Expression of Pseudomonas aeruginosa exoS is controlled by quorum sensing and RpoS. Microbiology 150, 843-851.

Hovey, A. K. \& Frank, D. W. (1995). Analyses of the DNA-binding and transcriptional activation properties of ExsA, the transcriptional activator of the Pseudomonas aeruginosa exoenzyme $\mathrm{S}$ regulon. J Bacteriol 177, 4427-4436.

Hueck, C. J. (1998). Type III protein secretion systems in bacterial pathogens of animals and plants. Microbiol Mol Biol Rev 62, 379-433.

Hung, D. T., Shakhnovich, E. A., Pierson, E. \& Mekalanos, J. J. (2005). Small-molecule inhibitor of Vibrio cholerae virulence and intestinal colonization. Science 310, 670-674.

Hwu, P., Du, M. X., Lapointe, R., Do, M., Taylor, M. W. \& Young, H. A. (2000). Indoleamine 2,3-dioxygenase production by human dendritic cells results in the inhibition of $\mathrm{T}$ cell proliferation. J Immunol 164, 3596-3599. 
Jain, M., Ramirez, D., Seshadri, R., Cullina, J. F., Powers, C. A., Schulert, G. S., Bar-Meir, M., Sullivan, C. L., McColley, S. A. \& Hauser, A. R. (2004). Type III secretion phenotypes of Pseudomonas aeruginosa strains change during infection of individuals with cystic fibrosis. J Clin Microbiol 42, 5229-5237.

Juhas, M., Eberl, L. \& Tummler, B. (2005). Quorum sensing: the power of cooperation in the world of Pseudomonas. Environ Microbiol 7, 459-471.

Kurnasov, O., Jablonski, L., Polanuyer, B., Dorrestein, P., Begley, T. \& Osterman, A. (2003). Aerobic tryptophan degradation pathway in bacteria: novel kynurenine formamidase. FEMS Microbiol Lett 227, 219-227.

Lambrecht, M., Okon, Y., Vande Broek, A. \& Vanderleyden, J. (2000). Indole-3-acetic acid: a reciprocal signalling molecule in bacteria-plant interactions. Trends Microbiol 8, 298-300.

Laskowski, M. A., Osborn, E. \& Kazmierczak, B. I. (2004). A novel sensor kinase-response regulator hybrid regulates type III secretion and is required for virulence in Pseudomonas aeruginosa. Mol Microbiol 54, 1090-1103.

Lee, V. T., Smith, R. S., Tummler, B. \& Lory, S. (2005). Activities of Pseudomonas aeruginosa effectors secreted by the type III secretion system in vitro and during infection. Infect Immun 73, 1695-1705.

Linares, J. F., Lopez, J. A., Camafeita, E., Albar, J. P., Rojo, F. \& Martinez, J. L. (2005). Overexpression of the multidrug efflux pumps MexCD-OprJ and MexEF-OprN is associated with a reduction of type III secretion in Pseudomonas aeruginosa. J Bacteriol 187, 1384-1391.

Liu, P. \& Nester, E. W. (2006). Indoleacetic acid, a product of transferred DNA, inhibits vir gene expression and growth of Agrobacterium tumefaciens C58. Proc Natl Acad Sci U S A 103, 4658-4662.

Loewen, P. C., Hu, B., Strutinsky, J. \& Sparling, R. (1998). Regulation in the rpoS regulon of Escherichia coli. Can J Microbiol 44, 707-717.

Lucchini, S., Liu, H., Jin, Q., Hinton, J. C. \& Yu, J. (2005). Transcriptional adaptation of Shigella flexneri during infection of macrophages and epithelial cells: insights into the strategies of a cytosolic bacterial pathogen. Infect Immun 73, 88-102.

Lyczak, J. B., Cannon, C. L. \& Pier, G. B. (2002). Lung infections associated with cystic fibrosis. Clin Microbiol Rev 15, 194-222.

Marx, C. J. \& Lidstrom, M. E. (2002). Broad-host-range cre-lox system for antibiotic marker recycling in Gram-negative bacteria. Biotechniques 33, 1062-1067.

McCaw, M. L., Lykken, G. L., Singh, P. K. \& Yahr, T. L. (2002). ExsD is a negative regulator of the Pseudomonas aeruginosa type III secretion regulon. Mol Microbiol 46, 1123-1133.

Morin, D., Grasland, B., Vallee-Rehel, K., Dufau, C. \& Haras, D. (2003). On-line high-performance liquid chromatography-mass spectrometric detection and quantification of $\mathrm{N}$-acylhomoserine lactones, quorum sensing signal molecules, in the presence of biological matrices. J Chromatogr A 1002, 79-92.

Nordfelth, R., Kauppi, A. M., Norberg, H. A., Wolf-Watz, H. \& Elofsson, M. (2005). Small-molecule inhibitors specifically targeting type III secretion. Infect Immun 73, 3104-3114.

Patten, C. L. \& Glick, B. R. (1996). Bacterial biosynthesis of indole-3acetic acid. Can J Microbiol 42, 207-220.

Patten, C. L. \& Glick, B. R. (2002). Role of Pseudomonas putida indoleacetic acid in development of the host plant root system. Appl Environ Microbiol 68, 3795-3801.

Pesci, E. C., Milbank, J. B., Pearson, J. P., McKnight, S., Kende, A. S., Greenberg, E. P. \& Iglewski, B. H. (1999). Quinolone signaling in the cell-to-cell communication system of Pseudomonas aeruginosa. Proc Natl Acad Sci U S A 96, 11229-11234.
Pier, G. B. (2002). CFTR mutations and host susceptibility to Pseudomonas aeruginosa lung infection. Curr Opin Microbiol 5, 81-86.

Pires de Melo, M., Curi, T. C., Miyasaka, C. K., Palanch, A. C. \& Curi, R. (1998). Effect of indole acetic acid on oxygen metabolism in cultured rat neutrophil. Gen Pharmacol 31, 573-578.

Pritchard, D. I. (2006). Immune modulation by Pseudomonas aeruginosa quorum-sensing signal molecules. Int $J$ Med Microbiol 296, 111-116.

Quenee, L., Lamotte, D. \& Polack, B. (2005). Combined sacB-based negative selection and cre-lox antibiotic marker recycling for efficient gene deletion in Pseudomonas aeruginosa. Biotechniques 38, 63-67.

Rietsch, A. \& Mekalanos, J. J. (2006). Metabolic regulation of type III secretion gene expression in Pseudomonas aeruginosa. Mol Microbiol 59, 807-820.

Rietsch, A., Wolfgang, M. C. \& Mekalanos, J. J. (2004). Effect of metabolic imbalance on expression of type III secretion genes in Pseudomonas aeruginosa. Infect Immun 72, 1383-1390.

Rietsch, A., Vallet-Gely, I., Dove, S. L. \& Mekalanos, J. J. (2005). ExsE, a secreted regulator of type III secretion genes in Pseudomonas aeruginosa. Proc Natl Acad Sci U S A 102, 8006-8011.

Roy-Burman, A., Savel, R. H., Racine, S., Swanson, B. L., Revadigar, N. S., Fujimoto, J., Sawa, T., Frank, D. W. \& Wiener-Kronish, J. P. (2001). Type III protein secretion is associated with death in lower respiratory and systemic Pseudomonas aeruginosa infections. J Infect Dis 183, 1767-1774.

Schaber, J. A., Carty, N. L., McDonald, N. A., Graham, E. D., Cheluvappa, R., Griswold, J. A. \& Hamood, A. N. (2004). Analysis of quorum sensing-deficient clinical isolates of Pseudomonas aeruginosa. J Med Microbiol 53, 841-853.

Schweizer, H. P. \& Hoang, T. T. (1995). An improved system for gene replacement and $x y l E$ fusion analysis in Pseudomonas aeruginosa. Gene $158,15-22$.

Shen, D. K., Filopon, D., Kuhn, L., Polack, B. \& Toussaint, B. (2006). PsrA is a positive transcriptional regulator of the type III secretion system in Pseudomonas aeruginosa. Infect Immun 74, 1121-1129.

Simon, R., Priefer, U. \& Puhler, A. (1983). A broad host range mobilization system for in vivo genetic engineering: transposon mutagenesis in Gram negative bacteria. Bio/Technology 1, 784-791.

Sperandio, V., Mellies, J. L., Nguyen, W., Shin, S. \& Kaper, J. B. (1999). Quorum sensing controls expression of the type III secretion gene transcription and protein secretion in enterohemorrhagic and enteropathogenic Escherichia coli. Proc Natl Acad Sci U S A 96, 1519615201.

Stover, C. K., Pham, X. O., Erwin, A. L., Mizoguchi, S. D., Warrener, P., Hickey, M. J., Brinkman, F. S., Hufnagle, W. O., Kowalik, D. J. \& other authors (2000). Complete genome sequence of Pseudomonas aeruginosa PAO1, an opportunistic pathogen. Nature 406, 959-964.

Terness, P., Bauer, T. M., Rose, L., Dufter, C., Watzlik, A., Simon, H. \& Opelz, G. (2002). Inhibition of allogeneic $T$ cell proliferation by indoleamine 2,3-dioxygenase-expressing dendritic cells: mediation of suppression by tryptophan metabolites. J Exp Med 196, 447-457.

Toussaint, B., Delic-Attree, I. \& Vignais, P. M. (1993). Pseudomonas aeruginosa contains an IHF-like protein that binds to the $\operatorname{alg} D$ promoter. Biochem Biophys Res Commun 196, 416-421.

Urbanowski, M. L., Lykken, G. L. \& Yahr, T. L. (2005). A secreted regulatory protein couples transcription to the secretory activity of the Pseudomonas aeruginosa type III secretion system. Proc Natl Acad Sci U S A 102, 9930-9935.

Vallis, A. J., Yahr, T. L., Barbieri, J. T. \& Frank, D. W. (1999). Regulation of ExoS production and secretion by Pseudomonas aeruginosa in response to tissue culture conditions. Infect Immun 67, 914-920. 
Ventre, I., Goodman, A. L., Vallet-Gely, I., Vasseur, P., Soscia, C., Molin, S., Bleves, S., Lazdunski, A., Lory, S. \& Filloux, A. (2006). Multiple sensors control reciprocal expression of Pseudomonas aeruginosa regulatory RNA and virulence genes. Proc Natl Acad Sci U S A 103, 171-176.

Vogel, H. J. \& Bonner, D. M. (1956). Acetylornithinase of Escherichia coli: partial purification and some properties. J Biol Chem 218, 97-106.

West, S. E., Schweizer, H. P., Dall, C., Sample, A. K. \& RunyenJanecky, L. J. (1994). Construction of improved EscherichiaPseudomonas shuttle vectors derived from pUC18/19 and sequence of the region required for their replication in Pseudomonas aeruginosa. Gene 148, 81-86.

Wolf, K., Betts, H. J., Chellas-Gery, B., Hower, S., Linton, C. N. \& Fields, K. A. (2006). Treatment of Chlamydia trachomatis with a small molecule inhibitor of the Yersinia type III secretion system disrupts progression of the chlamydial developmental cycle. Mol Microbiol 61, 1543-1555.

Wolfgang, M. C., Lee, V. T., Gilmore, M. E. \& Lory, S. (2003). Coordinate regulation of bacterial virulence genes by a novel adenylate cyclase-dependent signaling pathway. Dev Cell 4, 253-263.
Wong, S. M. \& Mekalanos, J. J. (2000). Genetic footprinting with mariner-based transposition in Pseudomonas aeruginosa. Proc Natl Acad Sci U S A 97, 10191-10196.

Woodward, A. W. \& Bartel, B. (2005). Auxin: regulation, action, and interaction. Ann Bot (Lond) 95, 707-735.

Yahr, T. L. \& Frank, D. W. (1994). Transcriptional organization of the trans-regulatory locus which controls exoenzyme $\mathrm{S}$ synthesis in Pseudomonas aeruginosa. J Bacteriol 176, 3832-3838.

Yahr, T. L. \& Wolfgang, M. C. (2006). Transcriptional regulation of the Pseudomonas aeruginosa type III secretion system. Mol Microbiol 62, 631-640.

Yanisch-Perron, C., Vieira, J. \& Messing, J. (1985). Improved M13 phage cloning vectors and host strains: nucleotide sequences of the M13mp18 and pUC19 vectors. Gene 33, 103-119.

Zolfaghar, I., Angus, A. A., Kang, P. J., To, A., Evans, D. J. \& Fleiszig, S. M. (2005). Mutation of retS, encoding a putative hybrid twocomponent regulatory protein in Pseudomonas aeruginosa, attenuates multiple virulence mechanisms. Microbes Infect 7, 1305-1316.

Edited by: P. Cornelis 